\title{
TEMA-2017: Broncoconstricción inducida por ejercicio y asma desencadenada por ejercicio
}
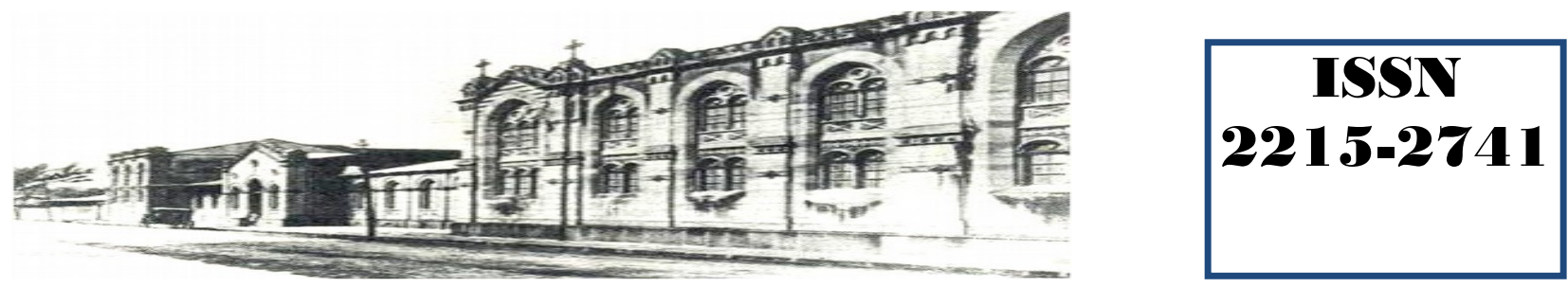

Hospital San quan de Dios, San Yosé. Costa Rica. Fundado en 1845

Recibido:

$10 / 03 / 2017$

Aceptado:

$15 / 04 / 2017$

Giovanni Sedó Mejía ${ }^{1}$

Gabriel Hernández Romero ${ }^{2}$

Gustavo Montero Solano ${ }^{3}$

Juan Carlos Vega Chaves ${ }^{4}$

${ }^{1}$ Médico Especialista en Medicina Interna y Alergología. Asistente del Servicio de Medicina Interna. Sección de Medicina, Hospital San Juan de Dios. Correo electrónico:

${ }^{2}$ Médico General. Universidad de Costa Rica. Correo electrónico: gabrielhdez93@gmail.com

${ }^{3}$ Medico General. Universidad de Costa Rica. Correo electrónico: gmonso@hotmail.com

${ }^{4}$ Médico General. Universidad de Costa Rica. Correo electrónico: juanca77vech@hotmail.com

\section{RESUMEN}

La broncoconstricción inducida por el ejercicio se refiere al estrechamiento transitorio de esta asociado al ejercicio. Se presenta con síntomas comunes como tos, sibilancias, opresión torácica y disnea. Una espirometría inicial debería realizarse para evaluar si existe asma subyacente. En un gran número de atletas se obtienen resultados normales. En este caso, se deberían realizar pruebas adicionales como la prueba de hiperpnea eucapnica voluntaria (HEV) o la prueba de ejercicio en campo deportivo o laboratorio. Los beta 2 agonistas de acción corta son los agentes de primera línea para tratamiento farmacológico. Los antagonistas de receptores de leucotrienos o los corticoesteroides inhalados con o sin beta 2 agonistas de acción larga pueden ser usados en casos persistentes.

\section{PALABRAS CLAVE}

Broncoconstricción. Ejercicio. Asma. Beta 2 agonistas. Corticoesteroides.

\begin{abstract}
Exercise-induced bronchoconstriction refers to the airway narrowing associated with physical activity. Common symptoms presented are coughing, wheezing, chest tightness and dyspnea. An initial spirometry should be performed to evaluate for underlying asthma. In a great num-
\end{abstract}


ber of athletes results are normal. In this case, additional tests such as eucapnica voluntary hyperpnea test or laboratory or field-based exercise challenge should be performed. Short-acting beta 2 agonists are the first-line agents for pharmacologic treatment. Leukotriene receptor antagonists or inhaled corticosteroids with or without longacting beta 2 agonists may be used persistent cases.

\section{KEY WORDS}

Bronchoconstriction. Exercise. Asthma. Beta 2 agonists. Corticosteroids.

\section{INTRODUCCIÓN}

La broncoconstricción inducida por el ejercicio (BIE) es una forma particular de hiperreactividad de la vía aérea, que hace referencia al estrechamiento transitorio de esta asociado al ejercicio, el cual es un fenómeno que ocurre frecuentemente en atletas que pueden tener o no el diagnóstico previo de asma. La hiperreactividad del árbol respiratorio, la cual es un elemento cardinal del asma, es más común en atletas de alta resistencia en comparación a la población general ${ }^{1}$. Es importante hacer distinción respecto a BIE y el término asma inducida por ejercicio (AIE). Este último es inadecuado dado que implica que el ejercicio causa asma, cuando en realidad es un desencadenante de síntomas que pueden ser controlados igual que otros factores. Además, la BIE no es exclusiva de pacientes con asma como lo sugiere el término EIA. La BIE en pacientes tanto asmáticos como no asmáticos presenta un traslape de síntomas considerable, mecanismos fisiopatológicos y tratamiento, por lo que tanto BIE como EIA se agrupan bajo el término BIE ${ }^{2}$. Más del $10 \%$ de la población general y hasta el $90 \%$ de los pacientes asmáticos cursan con BIE. La prevalencia de este trastorno en atletas es de 11 a $50 \%$, aunque se acerca al $90 \%$ en aquellos que cursan con el diagnóstico de asma. A su vez, ocurre con mayor frecuencia en atletas expuestos a clima frío, de manera que aproximadamente 50\% de los esquiadores olímpicos presentan $\mathrm{BIE}^{3,4}$.

\section{DISCUSIÓN}

\section{Fisiopatología}

No es la actividad física en sí la que desencadena el estrechamiento de la luz bronquial, sino que se postula que es la hiperpnea y el cambio térmico asociado y el acondicionamiento del aire inspirado (ver figura 1) ${ }^{5}$. Además, dado que la respiración oral es común durante el ejercicio, hay un aumento de penetración de alérgenos y agentes ambientales a las vías respiratorias inferiores, por ejemplo gases de cloro encontrados en piscinas techadas o gases liberados por pulidoras de hielo, contaminación ambiental como gases de combustión en regiones urbanas, o bien polen en campos abiertos $^{2,6}$.

Otro factor influyente es la injuria causada por el aire seco, el cual requiere uso de las vías aéreas más pequeñas para lograr condicionarse, causando daño en la capacidad contráctil del músculo liso ${ }^{2}$.

\section{Teoría Térmica}

Durante el ejercicio vigoroso, el calentamiento de grandes volúmenes de aire no acondicionado a temperatura corporal se asocia con la reducción en la temperatura de la vía aérea intratorácica e intrapulmonar ${ }^{2}$. Se ha propuesto desde 1986 que el enfriamiento de la hiperpnea durante el ejercicio causa vasoconstricción de la circulación bronquial. Posteriormente, al cesar el esfuerzo físico, se da una hiperemia reactiva que conlleva fuga vascular y edema en la mucosa y submucosa bronquial, causando el estrechamiento transitorio característico. En este escenario, el cuadro clínico se presentaría como consecuencia de eventos vasculares, sin asociarse a liberación de mediadores ${ }^{7}$.

\section{Teoría Osmótica}

La teoría osmótica contempla los efectos de la deshidratación de la vía aérea por la humidificación del aire inspirado. Se postula que el volumen de líquido en la superficie de la vía aérea es pequeño, por lo que el acondicionamiento del aire durante el ejercicio conlleva a deshidratación y aumento en la osmolaridad de la superficie de la vía aérea. Estos cambios conllevan al movimiento de agua de las células circundantes hacia el lumen de la vía aérea para restaurar el volu- 
men de agua perdido, lo cual causa contracción de las células subepiteliales. Consecuentemente, se liberan mediadores inflamatorios que causan broncoconstricción, como leucotrienos, prostaglandinas e histamina, durante el aumento de volumen compensador. En el contexto de una vía aérea hiperreactiva se presenta contracción de músculo liso ${ }^{5,6}$. Son elementales en esta respuesta los eosinófilos, mastocitos y células cilíndricas epiteliales. Esta teoría osmótica podría justificar por qué atletas competitivos tienen mayor incidencia de $\mathrm{EIB}{ }^{2,6}$. De esta manera, los deportes que más inducen BIE son los de mayor ventilación minuto (básquetbol, ciclismo, soccer) y aquellos que se dan en ambientes fríos (esquí de fondo, patinaje sobre hielo), los cuales se asocian a mayor deshidratación de la vía aérea tiende más a ocurrir. ${ }^{2}$

La teoría térmica no contempla múltiples elementos fundamentales de esta patología. Hay evidencia que muestra que el acondicionamiento del aire y recalentamiento no son eventos esenciales para desarrollar BIE, dado que estudios muestran que inspirar aire caliente, seco $\left(>37^{\circ} \mathrm{C}\right)$ puede causar BIE severa ${ }^{8}$. Otros estudios muestran que al dar a hiperventilar gases a diferentes temperaturas a individuos asmáticos el broncoespasmo puede ser inducido sin pérdida significativa de calor o sin enfriamiento significativo de la vía aérea. Esto sugiere que la BIE depende más de la pérdida de agua en las mucosas respiratorias 9 .

Otros hallazgos sugieren que la habilidad para transpirar está reducida en pacientes con reactividad bronquial aumentada. El epitelio glandular respiratorio y el epitelio glandular asociado a las glándulas sudoríparas comparten mecanismos de secreción de sodio, cloruro y agua dependiente del receptor muscarínico M3 idénticos. Consecuentemente, esto podría indicar que en individuos con BIE hay una alteración en la regulación de la homeostasis del líquido corporal ${ }^{10}$.

\section{Lesión de la vía aérea}

Al darse deshidratación de la vía aérea se compromete la integridad de la barrera epitelial, lo cual aumenta la posibilidad de que ocurra injuria celular. La hiperventilación de aire seco se asocia a descamación significativa del epitelio respira- torio, de manera que entre mayor sea el flujo ventilatorio y menor sea la humedad del aire, mayor es el daño. En asmáticos con BIE, muestras de esputos tomadas posteriores al ejercicio revelan daño al epitelio respiratorio significativo y concentraciones elevadas de celular epiteliales cílindricas, que correlacionan con liberación de mediadores respiratorios. Este daño a la barrera protectora podría causar que penetren elementos como bacterias, virus, alérgenos o irritantes que potencialmente causarían una reacción inflamatoria ${ }^{12}$. Otro componente importante es la lesión mecánica ejercida por fuerzas de cizalla, las cuales tienen su valor máximo durante la tos. De esta manera, la hiperpnea de aire frío y deshidratación del epitelio respiratorio activan nervios sensitivos y desencadenan la tos, la cual conlleva mayor inflamación de la vía aérea y remodelación. A pesar de esto, las fuerzas físicas ejercidas durante el ejercicio y el efecto de respiraciones profundas en individuos sanos tienen un efecto broncodilatador, no obstante, este muchas veces se ve anulado en pacientes asmáticos ${ }^{2}$.

Finalmente, se puede desarrollar inmunosupresión transitoria en atletas durante periodos de entrenamiento intenso, con aumento de susceptibilidad a infecciones, particularmente virales ${ }^{13}$. Aquellas personas con BIE y asma subyacente usualmente experimentan exacerbación de la inflamación subyacente e hiperreactividad de la vía aérea por cualquiera de los mecanismos mencionados o pobre control del asma crónica ${ }^{2,3}$.

\section{Diagnóstico}

Los síntomas típicos incluyen sibilancias, disnea, sensación de ahogo, tos y opresión torácica durante o posterior al ejercicio. Estos síntomas comúnmente ocurren durante actividad física intensa, hacen pico a los 5-10 minutos de haber finalizado y pueden durar desde 20 minutos hasta una hora. Si los síntomas se presentan rápidamente al iniciar la actividad física este diagnóstico es improbable, dado que al inicio hay una broncodilatación transitoria ${ }^{14,15}$. Entre los síntomas atípicos se incluye fatiga, sentirse físicamente fuera de forma, incapacidad de lograr el mismo ritmo de compañeros de ejercicio y malestar abdominal ${ }^{16}$. Los síntomas subjetivos han mostrado ser pobres predictores de BIE, dado su baja especificidad y que otras patologías como disfunción de cuerdas 
vocales, reflujo gastroesofágico, síndrome de hiperventilación, ansiedad o pobre condición física pueden asemejarse ${ }^{1,17,4}$. Otras patologías como arritmias, cardiomiopatías o trastornos anatómicos cardiopulmonares son más raros, pero deben tenerse en cuenta. El examen físico generalmente no muestra hallazgos relevantes. Si los pacientes se encuentran sintomáticos, lo más común es encontrar taquipnea y sibilancias espiratorias ${ }^{3}$.

En pacientes en los cuales se sospecha BIE se debería realizar una espirometría para identificar aquellos con función pulmonar alterada en reposo y descartar asma subyacente. Si se encuentran datos de obstrucción, se recomienda realizar pruebas adicionales antes y después del uso de albuterol. Es común que la espirometría en reposo de los pacientes con BIE sea normal. Para evitar falsos negativos hay que asegurarse que haya un adecuado esfuerzo físico y estrés ambiental ${ }^{15}$. Algunas fuentes recomiendan iniciar una prueba terapéutica, mientras que otras sugieren realizar más pruebas antes de dar tratamiento. Si el paciente no es un atleta de élite, se podría prescribir una prueba terapéutica con beta-agonistas de acción corta, se da seguimiento usualmente después de una a dos semanas, para determinar el éxito del tratamiento y en caso de respuesta inadecuada se realizan más pruebas. Para atletas de élite o individuos cuya respuesta al beta-agonista de acción corta no fue óptima, se usan pruebas de provocación bronquial, ya sean directas o indirectas, para identificar caídas en el volumen espiratorio forzado en el primer segundo (VEF1). Si las pruebas de provocación bronquial fueran normales, se deberían considerar otros diagnósticos 3,15 .

Una prueba de broncoprovocación positiva indica que hay necesidad de tratamiento. Una caída $\geq$ $10 \%$ o más en el VEF1 antes y después de la prueba es el criterio usado para el diagnóstico. Se prefiere VEF1 como parámetro respecto al flujo espiratorio máximo, dado que es un valor más reproducible y discriminatorio. La medición se realiza antes de la prueba y de manera seriada después del reto determinado, hasta 30 minutos después, utilizando como parámetro el valor más bajo ${ }^{15,17}$. Las pruebas indirectas pueden ser realizadas con un espirómetro portátil en el sitio en el cual el paciente desarrolla la sintomatología. Estas tienen mayor sensibilidad y por tanto son pre- feridas para hacer el diagnóstico de BIE. Sin embargo, es necesario realizar más estudios para determinar si alguna prueba se podría considerar estándar de oro para el diagnóstico. A continuación se describirá brevemente cada una de ellas ${ }^{3}$.

\section{Prueba de hiperpnea eucapnica vo-} luntaria (HEV)

El Comité Olímpico Internacional recomienda esta prueba como herramienta diagnóstica de elección para BIE en los competidores. El paciente hiperventila durante 6 minutos una mezcla de aire al 5\% de dióxido de carbono y $21 \%$ de oxígeno. Ha mostrado tener alta especificidad, sin embargo no está disponible ampliamente ${ }^{15,17}$.

Prueba de ejercicio en campo deportivo

Se realiza una espirometría antes y después de desarrollar la actividad física que desencadena los síntomas en el paciente, por tanto es deporteespecífica. Es menos sensible que la $\mathrm{HEV}^{1,15}$.

Nebulización con solución salina hipertónica

Se realiza una espirometría antes y después de nebulizar al paciente con una solución salina al $4,5 \%$. No es tan confiable si el paciente está bajo tratamiento con corticosteroides inhalados. Correlaciona con la severidad de la BIE ${ }^{15,17}$.

\section{Prueba de ejercicio en laboratorio}

Es la prueba más estudiada y está estandarizada. Requiere que se realice a una intensidad lo suficientemente vigorosa para evitar falsos negativos. Se realiza espirometría antes y después de una actividad física, generalmente en bicicleta estacionaría o máquina caminadora. Como no se realiza en las condiciones específicas en las que se desarrolla el cuadro, no es deporte-específica 15,18 .

\section{Prueba de inhalación de manitol}

Consiste en administrar manitol inhalado a dosis crecientes para crear un efecto osmótico en la vía aérea. La prueba termina cuando se administra la última dosis de manitol (hasta $160 \mathrm{mg}$ ) o al caer 
el VEF1 en $15 \%$ o más. Es un test portátil y se puede hacer en campo o en laboratorio. Una de sus limitaciones es que provoca tos, lo cual puede causar falsos negativos al disminuir la dosis depositada en la vía aérea inferior. No es tan sensible en individuos asmáticos bajo tratamiento con corticoesteroides inhalados ${ }^{15,17}$.

\section{Manejo}

La meta principal del tratamiento es permitir que el paciente realice actividad física sin riesgo. Se busca lograr que el atleta mantenga el nivel competitivo deseable y ayudarlo a lograr su mayor capacidad ${ }^{19,3}$. Entre los tratamientos no farmacológicos se incluye evitar agentes gatillo y escoger deportes con baja ventilación minuto. A pesar de que algunas opciones no farmacológicas pueden ser efectivas, todos los atletas con BIE necesitan beta-agonistas de acción corta. Existe evidencia que el calentamiento previo a la actividad física puede atenuar la BIE, al inducir un periodo refractario. Sin embargo, esto no ha mostrado ser efectivo en atletas de élite de ambientes fríos. Otra opción son las máscaras de intercambio de calor las cuales están diseñadas para limitar la exposición a aire frio. El uso de estas no ha mostrado ser tan efectivo como el pretratamiento con albuterol, además de que no son prácticas durante competencias ${ }^{1,3}$. Otros abordajes incluye la restricción dietética de sodio por una o dos semanas, sin embargo no hay estudios a largo plazo. Un estudio pequeño, doble ciego, mostró que altas dosis de aceite de pescado con omega-3 por 3 semanas redujo el uso de broncodilatadores. Sin embargo, era una muestra pequeña y no se hizo mención a efectos adversos a la dosis utilizada ${ }^{20}$.

Respecto a tratamiento farmacológico, esta es la base del manejo de los pacientes con BIE. A continuación se describen las diferentes opciones terapéuticas.

\section{Beta 2 Agonistas Inhalados}

Los de acción corta se recomiendan como opción de primera línea de tratamiento. Se recomienda usarlos 15 minutos antes de la actividad física. Típicamente tienen un pico de acción a los 15-60 minutos, y la duración de su efecto es aproximadamente 3 horas. Dado que hay desarrollo de taquifilaxia asociada a su uso continuo, se deberían usar solo antes de actividad física intensa o antes de una competencia, de lo contrario, se reduce el beneficio en la prevención de BIE. Por tanto, en aquellos que realizan ejercicio diario o requieren el uso constantes de estos, se debe recurrir a otras líneas terapéuticas ${ }^{1,21}$. Respecto a los de acción prolongada, se recomienda que no se usen en personas con asma a menos que haya uso concomitante de medicamentos para el control de su patología, como los son los corticoesteroides inhalados. El uso concomitante de corticoesteroides inhalados y beta agonistas inhalados de larga duración ha mostrado ser superior y efectivo al uso de corticoesteroides como monoterapia en el manejo de $\mathrm{BIE}^{22}$.

\section{Estabilizadores de mastocitos}

Estos han mostrado ser más efectivos que los anticolinérgicos pero menos efectivos que los beta-agonistas de acción corta en el manejo de BIE. Se deben utilizar de 15 a 20 minutos antes de iniciar la actividad física ${ }^{1}$.

\section{Corticoesteroides inhalados}

Son la piedra angular en el control de pacientes asmáticos. Un meta-análisis mostró que el uso de estos medicamentos por 4 semanas o más reduce el porcentaje de caída del FEV1 posterior a la actividad física. Se ha visto que el uso continuo induce una reducción progresiva en la respuesta a varios estímulos en la vía aérea, incluido el ejercicio. No reducen la tolerancia a los beta 2 agonistas 22 .

Antagonistas de receptores de leucotrienos

Han mostrado tener un beneficio persistente en el BIE. Se consideran terapia de segunda línea. Montelukast tiene un tiempo de inicio de acción de 2 horas y un beneficio preventivo continuado de hasta 24 horas después de una sola dosis oral. Comparado con salmeterol, montelukast es más efectivo a las 24 horas. A pesar de esto, es inferior al beta-agonista de acción corta. Montelukast no ha mostrado causar taquifilaxia ${ }^{23,24}$.

\section{Otros agentes}

Ipatropium (Atrovent) es un anticolinérgico que 
provee cierta protección contra la BIE pero no es tan efectivo como el beta-agonista de acción corta o el antagonista de receptores de leucotrienos. Otros como la heparina inhalada y la furosemida han mostrado ser efectivos, sin embargo solo se han hecho estudios con muestras pequeñas ${ }^{3}$.

Manejo a nivel institucional nacional (Caja Costarricense del Seguro Social)

De acuerdo a la Lista Oficial de Medicamentos de la CCSS vigente, se dispone de salbutamol como beta 2 agonista inhalado de acción corta y con fumarato de formoterol como beta 2 agonista inhalado de acción prolongada, de uso exclusivo de alergología, inmunología, neumología y medicina interna. Además se cuenta con dipropionato de beclometasona como corticoesteroide inhalado y con montelukast como antagonista de receptores de leucotrienos, de uso exclusivo de alergología, inmunología, neumología y medicina interna. De esta manera, se cuenta con las opciones terapéuticas de primera línea, así como alternativas. Por tanto, un manejo apropiado consistiría en el uso inicial de salbutamol pre-ejercicio de manera ocasional. Una alternativa disponible, menos eficaz respecto al salbutamol, corresponde a montelukast, que podría ser útil en aquellos que no presentan una respuesta adecuada o prefieren la vía oral sobre la inhalada. En aquellos pacientes quienes no responden o requieren uso frecuente de salbutamol se recomienda iniciar un esteroide inhalado como beclometasona. Respecto a fumarato de formoterol, no se recomienda su uso como monoterapia, por lo que su uso sería conveniente en pacientes quienes ameritan escalonamiento de la terapia por falta de respuesta o mal control en pacientes asmáticos, con el uso de salbutamol y beclometasona ${ }^{25}$.

\section{CONCLUSIONES}

La broncoconstricción inducida por ejercicio es una patología cada vez más estudiada, dada su alta prevalencia, no solo en pacientes asmáticos, sino que también en atletas de alto rendimiento. La fisiopatología tiene múltiples elementos que están interrelacionados, con la teoría osmótica siendo la más aceptada. De esta manera, la hiperpnea, el cambio térmico y la deshidratación por acondicionamiento del aire inspirado, con daño concomitante de la vía aérea y penetración de alérgenos e irritantes son los factores primordiales.

El diagnóstico depende de las pruebas de broncoprovocación indirectas, de las cuales no hay estudios que apunten hacia un estándar de oro. El manejo depende de los beta 2 agonistas como primera línea. Sin embargo debe usarse concomitante con control de la patología subyacente en asmáticos y uso de beta 2 agonistas de acción larga y esteroides en aquellos que requieran más dosis o realicen actividad física diaria. Otras opciones son los antagonistas de receptores de leucotrienos y anticolinérgicos.

\section{BIBLIOGRAFÍA}

1. Boulet L, O'Byrne P. Asthma and exercise-induced Bronchoconstriction in Athletes. N. Engl. J. Med. 2015; 372:641-648.

2. Craig T, Dispenza M. Benefits of exercise in asthma. Ann Allergy Asthma Immunol. 2013; 110(3):133-140.

3. Krafczyk M, Asplund C. Exercise-Induced Bronchoconstriction: Diagnosis and Management. Am Fam Physician. 2011. 84(4):427-434.

4. Weiss P, Rundell KW. Imitators of exercise-induced bronchoconstriction. Allergy Asthma Clin Immunol. 2009; 5(1):7-15

5. Kippelen P, Anderson S. Pathogenesis of exercise-induced bronchoconstriction. Immunol Allergy Clin North Am. 2013; 33: 299-312.

6. Pongdee T, Li J. Exercise-induced bronchoconstriction. Ann Allergy Asthma Immunol. 2013; 110(5): 311-315

7. McFadden ER, Lenner KA, Strohl KP. Postexertional airway rewarming and thermally induced asthma. New insights into pathophysiology and possible pathogenesis.J Clin Invest 1986; 78:1825.

8. Aitken ML, Marini JJ. Effect of heat delivery and extraction on airway conductance in normal and in asthmatic subjects. Am Rev Respir Dis. 1985; 131:357e361.

9. Strauss RH, McFadden ER, Ingram RH, et al. Influence of heat and humidity on the airway

10. Argyros GJ, Phillips YY, Rayburn DB, et al. Water loss without heat flux in exercise-induced bronchospasm. Am Rev Respir Dis. 1993;147:1419e1424. 
11. Park C, Stafford C, Lockette W. Exercise-induced asthma may be associated with diminished sweat secretion rates in humans. Chest 2008;134:552-8.

12. Hallstrand TS, Moody MW, Wurfel MM, et al. Inflammatory basis of exercise induced bronchoconstriction. Am J Respir Crit Care Med 2005;172:679-86.

13. Walsh P, Gleeson M, Shephard J, et al. Position statement. Part one. Immune function and exercise. Exercise Immunology Review. 2011;17:663.

14. Mayers L, Rundell K, Im J et al. Self-reported symptoms and exercise-induced asthma in the elite athlete. Med Sci Sports Exerc. 2001;33(2):208-213.

15. Parsons J. Exercise-induced Bronchoconstriction. Otolaryngol Clin N Am. 2014; 47:119-126.

16. De Baets F, Bodart E, Dramaix-Wilmet $M$ et al. Exercise-induced respiratory symptoms are poor predictors of bronchoconstriction. Pediatr. Pulmonol. 2005; 39: 301-305.

17. Parsons J, Hallstrand S, Mastronarde J et al. An official American Thoracic Society clinical practice guideline: exercise-induced bronchoconstriction. Am J Respir Crit Care Med. 2013; 187(9):1016-1027.

18. Weiler J, Hallstrand T, Parsons J et al. Improving screening and diagnosis of exercise-induced bronchoconstriction: a call to action. J Allergy Clin Immunol Pract. 2014; 2(3):275-280.

19. Backer V, Sverrild A, Porsjberg C. Treatment of Exercise-Induced Bronchoconstriction. Immunol Allergy Clin North Am. 2013; 3: 347-362.

20. Mickleborough T, Lindley M, Ionescu A, Fly A. Protective effect of fish oil supplementation on exercise-induced bronchoconstriction in asthma. Chest. 2006; 129(1):39-49.

21. Hancox R, Subbarao P, Kamada D, Watson R, Hargreave F, Inman M. Beta2-agonist tolerance and exercise-induced bronchospasm. Am J Respir Crit Care Med. 2002; 165:1068-1070.

22. Koh MS, Tee A, Lasserson TJ, Irving LB. Inhaled corticosteroids compared to placebo for prevention of exercise induced bronchoconstriction. Cochrane Database Syst Rev. 2007; (3):CD002739.

23. Peroni D, Pescollderungg L, Sandri $M$ et al. Time-effect of montelukast on protection against exercise-induced bronchoconstriction. Respir Med. 2011; 105:1790-1797.

24. Philip G, Pearlman D, Villarán C, et al. Single- dose montelukast or salmeterol as protection against exercise-induced bronchoconstriction. Chest. 2007; 132(3):875-883.

25. Caja Costarricense de Seguro Social. Gerencia Médica. Dirección de Farmacoepidemiología. Lista Oficial de Medicamentos. 2014. 


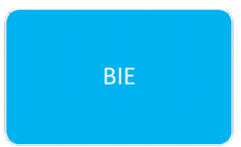

Figura 1. Fisiopatología

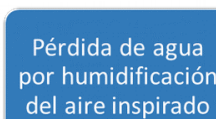

del aire inspirado

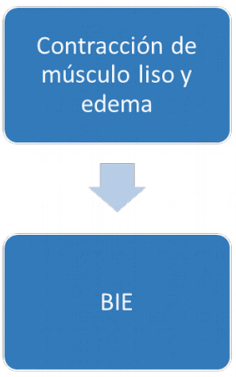

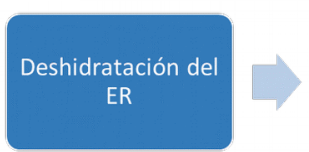

Contracción celular y liberación de mediadores
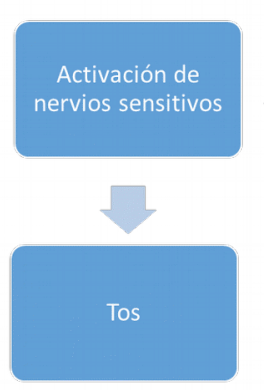

Movimiento de

agua para

restaurar la

superficie del ER
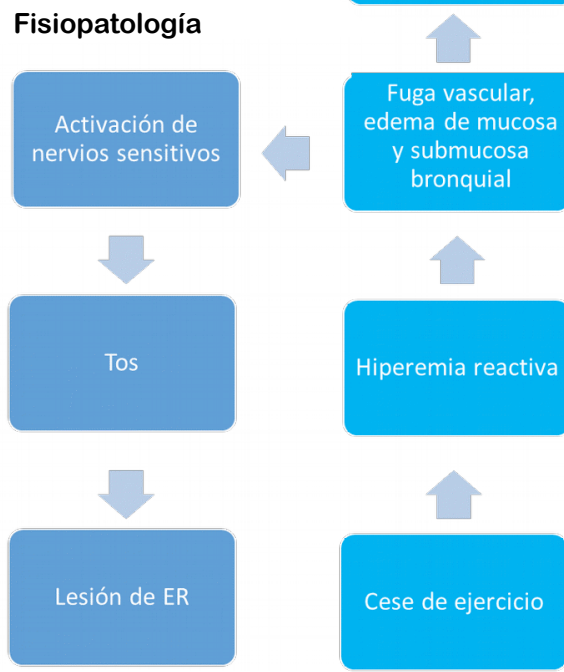

Acondicionamient

o de aire inspirado

y pérdida de calor

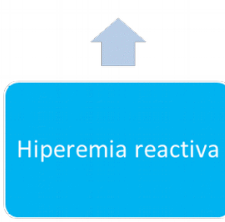

Enfriamiento

asociado a

hiperpnea

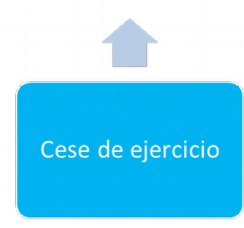

Vasconstricción en

circulación

bronquial

de la Broncoconstricción inducida por asma. ER: epitelio respiratorio

Fuente: Propia 